\title{
Detector Part of the Station for the Research and Irradiation of Promising Products of Semiconductor Micro- and Nanoelectronics with High-Energy Ion Beams
}

\author{
O. A. Vasil'ev ${ }^{a}$, A. G. Voronin ${ }^{a}$, D. E. Karmanov ${ }^{a}$, I. M. Kovalev ${ }^{a}$ * , A. A. Kurganov ${ }^{a}$, \\ M. M. Merkin ${ }^{a}$, A. D. Panov ${ }^{a}$, D. M. Podorozhnyi ${ }^{a}$, A. A. Slivin ${ }^{b}$, E. M. Syresin ${ }^{b}$, \\ A. N. Turundaevskii ${ }^{a}$, and G. A. Filatov ${ }^{b}$ \\ ${ }^{a}$ Skobeltsyn Institute of Nuclear Physics, Moscow State University, Moscow, 119234 Russia \\ ${ }^{b}$ Joint Institute for Nuclear Research, Dubna, 141980 Russia \\ *e-mail:im.kovalev@physics.msu.ru
}

Received August 15, 2020; revised October 27, 2020; accepted November 2, 2020

\begin{abstract}
In this paper, the authors present the composition of a prototype of the Detector Part of the Station (DPS) that uses a novel method for studying the integrated circuit durability to single-event effects (SEEs) during operation in outer space. The prototype operability is verified using Monte Carlo simulation.
\end{abstract}

DOI: $10.1134 / \mathrm{S} 1547477121020205$

\section{INTRODUCTION}

Complex large-scale integrated circuits (LSICs) operating in outer space are exposed to the influence of solar radiation and galactic particles. The higher the speed of the LSICs is, the more vulnerable they are to these influences. Modern LSICs are composed of submicron-sized elements, which means that they have smaller node capacities and, consequently, smaller charges for information storage. Lower voltages and powers also mean lower charges and currents needed to store and change information. All these factors make the devices more sensitive to radiation and mean that even low-energy particles can cause failures. Two classes of radiation effects have to be considered when creating specialized LSICs operating in the conditions of outer space [1]:

(i) total ionizing dose (TID);

(ii) single-event effects (SEEs).

The accumulated dose of ionization causes slow degradation of the integrated circuits (ICs) due to the charge accumulated in the chip material. SEEs are caused by single high-energy ionizing particles and their impact is intermittent; single particles occasionally reach sensitive microvolumes of the chip memory elements and induce, in particular, logic state switchover [1].

SEEs can be divided into reversible and irreversible [2]. Reversible switchovers (errors) usually occur in static and dynamic memory storage devices and mean a reversible switching of a cell from one state to another without any loss in the cell operability (single- event upset (SEU)). There have long been effective error detection and correction (EDAC) methods that can be implemented at both hardware and software levels.

Single events leading to irreversible destructive consequences are much more dangerous. The main types of such catastrophic failures are single-event latchup (SEL), single-event burnout (SEB) of highpower MOSFETs, and transistor oxide breakdown by a single particle (single-event gate rupture (SEGR)).

\section{SPECIFICITY OF STUDIES OF DURABILITY TO SINGLE EVENT EFFECTS}

Nowadays, two cumulative methods are used to study the IC durability to SEEs: the low-energy heavy nucleus beam method and pulsed laser method [3-7]. Both approaches have certain advantages and disadvantages $[8,9]$. It is important to note that the ion accelerators operating in Russia (in particular, U-400 and U-400M [10]) produce rather a wide beam, usually larger than the size of the sample under test, which allows one to study only the sensitivity parameters averaged over the entire sample. However, it is obvious that threshold linear energy losses (LELs) and cross sections of different parts (units) of modern specialized LSICs can vary greatly.

The use of a laser simulator with a focused spot a few micrometers in diameter $[7,9]$ and a comparable accuracy of its aiming allows us to circumvent this difficulty, but there are some other problems. The most significant of them is related to the presence of multi- 


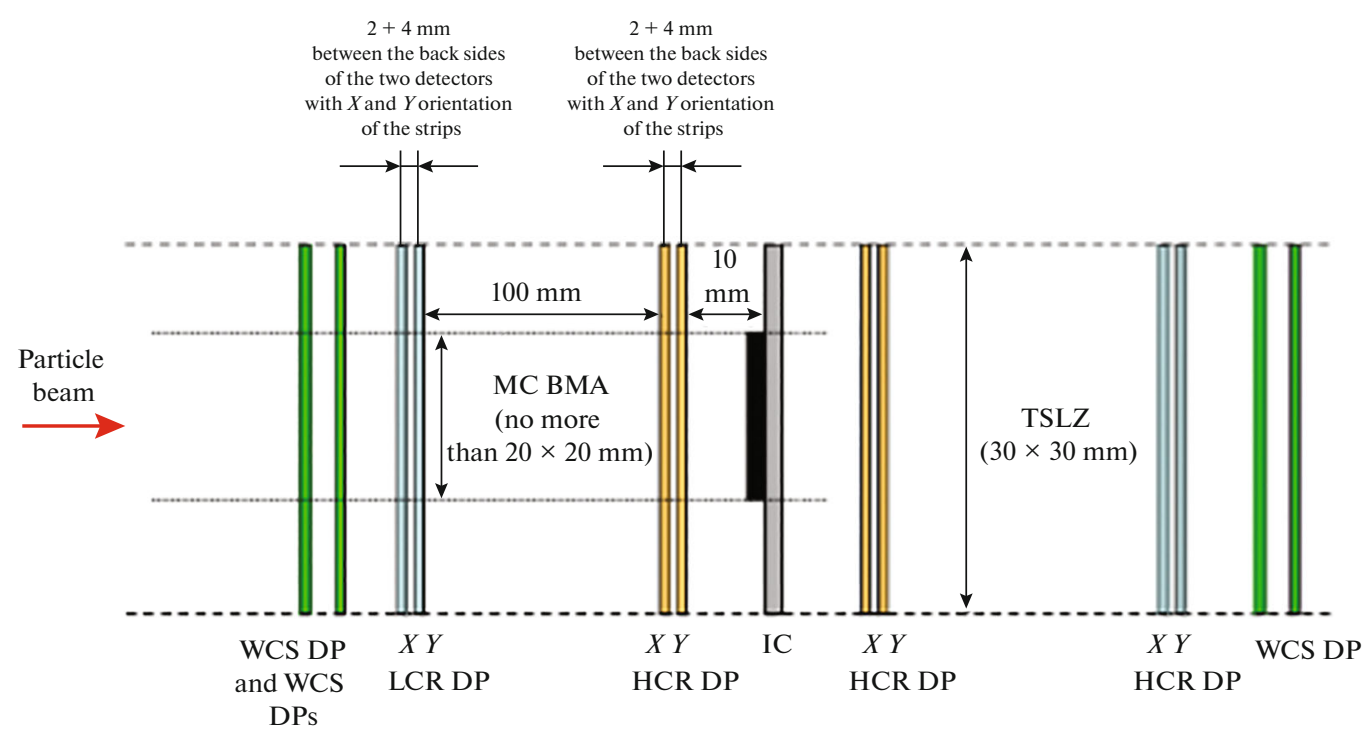

Fig. 1. Layout of the plane arrangement in the DPS.

layer metallization on the surface of modern specialized LSICs, which covers a significant part of their area. This can lead to "blank shots" of laser radiation, when optical radiation is almost completely reflected from metallization layers in certain parts of the sample [8], which obviously distorts the measured value of the SEE cross sections. However, even if the radiation penetrates through the protective layers, its LELs are underestimated compared to the real ionizing particle, which can lead to systematic errors in determining the threshold LELs.

These problems can be solved by combining the study on laser simulators with the study on ion accelerators by supplying the latter with the equipment that makes it possible to recover the point of the accelerator particle entry into the sample. This study does not replace tests with a laser simulator, since data acquisition using an ion accelerator is very expensive, especially considering that a wide range of LELs should be measured to investigate different types of SEEs [8]. However, the coordinate referencing of the accelerator tests allows calibrating data from the laser simulator by measuring, for example, systematic shifts in critical LEL values.

The equipment necessary for such studies is well known in high-energy physics: the so-called track systems.

\section{DETECTOR PART OF THE STATION}

The Detector Part of the Station (DPS) is an experimental facility that allows recovering trajectories (tracks) and energies (by energy release in the layers) of the particles entering it.

The DPS consists of several detecting planes (DPs) arranged one after another perpendicular to the accel- erator beam (the plane arrangement in the facility is presented in Fig. 1).

There are three types of planes used in the system:

(i) detecting planes with high coordinate resolution (HCR DPs);

(ii) detecting planes with low coordinate resolution (LCR DPs);

(iii) detecting planes without coordinate sensitivity (WCS DPs).

All types of DPs have the same shape: centrally symmetric relative to the accelerator beam axis, regardless of the type of detector installed. The sensitive element, silicon detector, is located at the center of the plane. The method of DP mounting in relation to the beam is the same for all types of the planes.

The DPS also includes units that are not mounted directly under the accelerator beam:

(i) a unit of controller boards and data-reading start-signal generation board (controller unit (CU));

(ii) the DPS control computer communication unit (CCU), a commercially available product);

(iii) the necessary supply units (commercially available products).

The abovementioned units are located outside the accelerator beam, but in close proximity to it (at a distance of $1-2 \mathrm{~m}$ ).

The DPS also includes the control computer with specialized DPS software, including:

(i) DPS part management software;

(ii) data preprocessing software.

The DPS control computer is located in the room of the operators.

The DPS provides the necessary measurements within the so-called tested sample location zone 
(TSLZ), which is $30 \times 30 \mathrm{~mm}$ in size. At the same time, the tested microchip body maximum area (MC BMA) should not exceed $4 \mathrm{~cm}^{2}$ within the TSLZ.

The expected range of the so-called linear energy losses (LELs) due to nuclei in the microchip is 1$80 \mathrm{MeV} \mathrm{cm}^{2} / \mathrm{mg}$ (which for silicon ICs corresponds to the energy release of $\sim 0.2-20 \mathrm{MeV} / \mu \mathrm{m})$. For these LEL values, the expected signal level in the DPS silicon sensors ranges from $0.13 \mathrm{pC}$ (for relativistic carbon nuclei) to $300 \mathrm{pC}$ (for slow gold nuclei) for a sensor thickness of $300 \mu \mathrm{m}$. Within these limits, the DPS provides an accuracy of determining the energy release (energy loss) in the microchip that is no worse than $10 \%$ and an accuracy of determining the point of the nucleus entry into the microchip that is no worse than $\pm 100 \mu \mathrm{m}$.

The DPS allows recording the energy-release value and the coordinates of entry of an individual nucleus into the microchip body for at least $65 \%$ of nuclei entering the MC BMA (i.e., $4 \mathrm{~cm}^{2}$ ) at nucleus fluxes not exceeding $10^{4} \mathrm{n} / \mathrm{s}$ (this corresponds to a flux of $2.2 \times 10^{4} \mathrm{n} / \mathrm{s}$ per entire TSLZ totaling $9 \mathrm{~cm}^{2}$ ). This evaluation of the recording efficiency is obtained under the assumption that the time of an event recording in DPS does not exceed $40 \mu$ s (the so-called dead time of the system in the full event-processing mode). However, this time is sufficient only for the events which include a single particle (projectile nucleus) which does not undergo nuclear transformations (interactions) in the DPS sensors. Events with the decay of the original nucleus are recorded more slowly and lead to additional losses in event recording.

The DPS makes it possible to count the number of nuclei hitting the IC body with a loss of no more than $5 \%$ for nucleus fluxes of no more than $10^{4} \mathrm{n} / \mathrm{s}$ per MC BMA. For higher fluxes, the statistic losses in the counting mode increase to no more than $30 \%$ for a flux of $10^{5} \mathrm{n} / \mathrm{s}$ per TSLZ $\left(9 \mathrm{~cm}^{2}\right)$, which is determined by the DPS dead time in the counting mode, which is less than $4 \mu$ s when counting the events with energy release above a certain threshold.

One silicon detector is located on each detection plane.

A "fine-stripped" detector installed on the HCR DP has the following characteristics: strip spacing $100 \mu \mathrm{m}, 320$ strips, detector dimensions of $34.0 \mathrm{~mm}$ (across strips) $\times 32.4 \mathrm{~mm}$ (along strips), and detector thickness of $300 \mu \mathrm{m}$.

A "large-stripped" detector installed on the LCR DP has the following characteristics: strip spacing $1 \mathrm{~mm}, 32$ strips, detector dimensions $34.0 \mathrm{~mm}$ (across strips) $\times 32.4 \mathrm{~mm}$ (along strips), and detector thickness $300 \mu \mathrm{m}$.

A "pad" detector installed on WCS DP is not divided into parts (strips); its dimensions are $34.0 \times$
$32.4 \mathrm{~mm}$ and its thickness is $300 \mu \mathrm{m}$. The pad detectors used in the pDPS are of two types: large and small. The dimensions and thicknesses of the large and small detectors are the same, $34.0 \times 32.4 \mathrm{~mm}$ and $300 \mu \mathrm{m}$, respectively, while their active areas differ in sizes.

Specialized integrated microcircuits NUKLON (NUKLON ASIC (Application-Specific Integrated Circuit)) are mounted on HCR DP and LCR DP for channel reading; these microcircuits showed good results in the successful NUKLON space experiment [11]. NUKLON ASIC is a 32-channel microchip for reading signals from silicon detectors with an unprecedentedly wide dynamic range of perceived signals.

The active area of all detectors except for the small WCS detector is the same, $32 \mathrm{~mm}$ across the strips (if any) and $29.6 \mathrm{~mm}$ along the strips (if any). The active area dimensions of the small WCS detector are $20.0 \times$ $20.0 \mathrm{~mm}$ and its thickness is $300 \mu \mathrm{m}$. The center of the active area of the small WCS detector coincides with the center of its overall dimensions.

The operation of the recording part of the DPS is controlled by two units: the controller unit (CU) and the computer communication unit (CCU).

The CCU unit is a standard Ethernet hub (commercially available) with at least 16 inputs. The CCU exchange rate with the DPS parts and with the control computer is $5-10 \mathrm{MB} / \mathrm{s}$. The CCU is connected to the DPS control computer, to all DPS planes (via intermediate CU controller boards), and to the trigger controller board within the $\mathrm{CU}$, allowing them to pass information between each other.

\section{MATHEMATICAL MODELING OF THE METHODS OF OPERATION}

This facility can operate in two modes of operation: transit-time mode and fixed-LEL mode.

The transit-time mode is a preliminary mode designed for carrying out the station calibration and studying the structure of the examined sample. This mode makes it possible, among other things, to localize the crystal inside the body of the microchip under study.

The fixed-LEL mode is the main mode designed to investigate the fault and failure resistance of the microchip under testing when hit by particles with the given LEL value. The energy of the projectile particles is selected based on the results of the "transit-time" data analysis, so that they leave a certain LEL in the microchip. The DPS sensors record the energy release and the coordinates of the projectile nuclei.

Figure 2 gives a general idea of the operation principle of the energy tomography technique. The figure shows two three-dimensional diagrams of the energy release by gold nuclei in the first (left diagram) and second (right diagram) pad planes downstream from the sample in the microchip with air voids. The $X$ and 

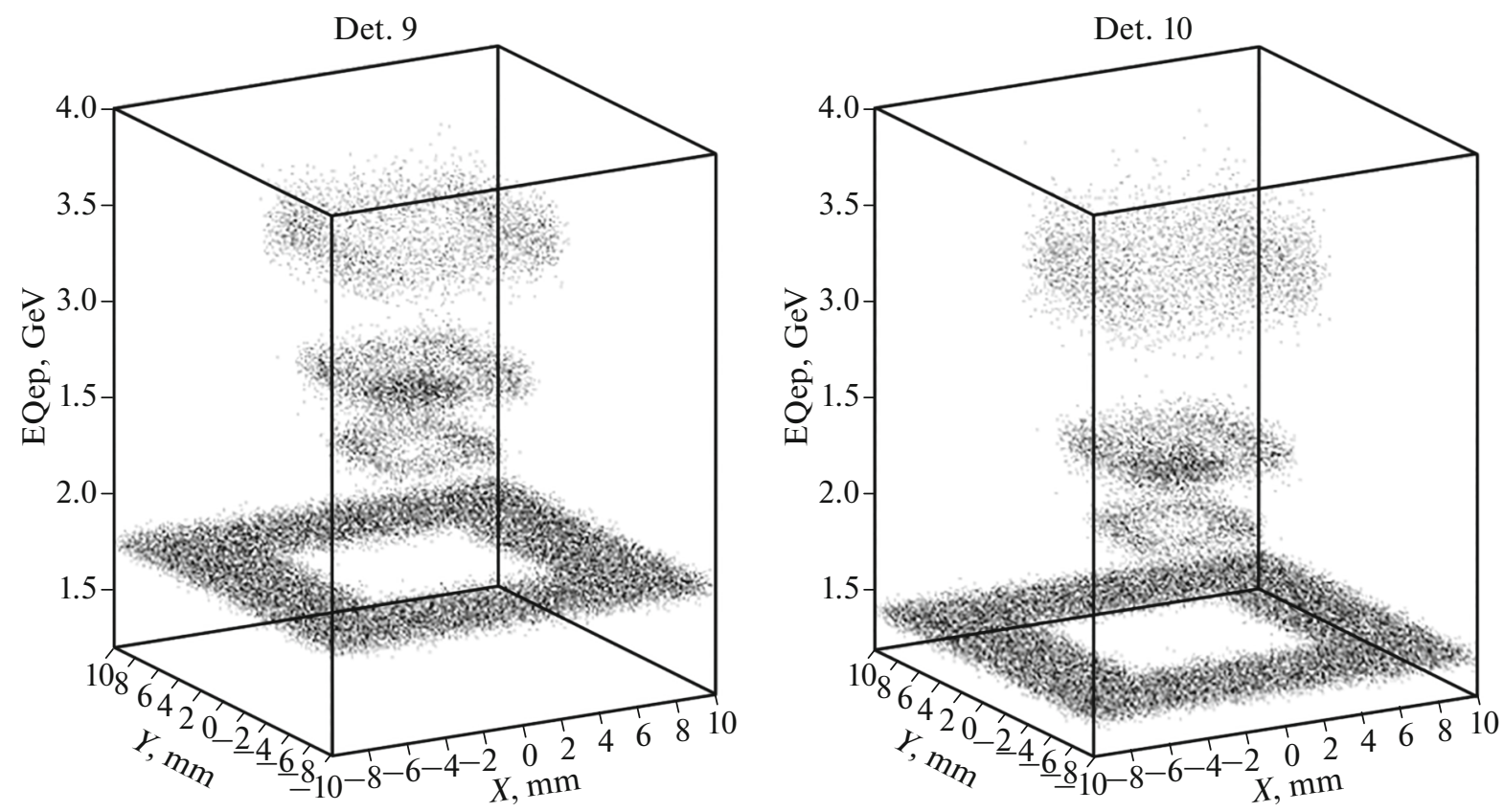

Fig. 2. Energetic tomography of the microchip model in a ceramic body (with air voids).



Fig. 3. Distribution of deviations of the recovered coordinates of particle tracks from the point of their entry into the microchip in the "fixed LEL" mode.

$Y$ axes (millimeters) show the recovered coordinates of the nucleus as it passes through the silicon microchip. The $Z$ axis shows the energy release in $\mathrm{GeV}$. One can clearly see how the energy release resolves the basic structures inside the microchip.

An example of error distribution for one of the transverse coordinates at the energy of $205 \mathrm{MeV} / \mathrm{n}$ is shown in Fig. 3. The standard deviation of the recovered coordinate from the impact coordinate at this energy is $50 \mu \mathrm{m}$. Within the simulation, it was shown that the obtained coordinate resolution weakly depended on the energy and did not exceed $56 \mu \mathrm{m}$ in the worst case [12].

\section{CONCLUSIONS}

A new technique for studying the durability of integrated circuits to single-event effects has been developed. A prototype of the DPS is developed to implement this technique. The Monte Carlo simulations that were performed have clearly proven the character- 
istics provided by the prototype. The prototype is currently under construction.

\section{FUNDING}

This paper was supported by the Ministry of Science and Higher Education of the Russian Federation; the unique identifier of the agreement is RFMEFI60719X0326.

\section{REFERENCES}

1. K. I. Tapero, V. N. Ulimov, and A. M. Chlenov, Radiation Effects in Silicon Integrated Circuits for Space Applications (BINOM, Labor. Znanii, 2012), p. 304.

2. Single Event Effects Specification. https://radhome.gsfc.nasa.gov/radhome/papers/seespec.htmhttps://radhome.gsfc.nasa.gov/radhome/papers/seespec.htm.

3. A. I. Chumakov, The Effect of Space Radiation on the IC (Radio Svyaz', Moscow, 2004) [in Russian].

4. G. R. Allen, "Compendium of test results of single event effects conducted by the jet propulsion laboratory," in Proceedings of the 2008 IEEE Radiation Effects Data Workshop.

5. V. Pouget, "Fundamentals of laser SEE testing and recent trends," in Proceedings of the RALFDAY, EADS, France, Suresnes, Sept. 11, 2009.

6. R. Jones et al., "Comparison between SRAM SEE cross-section from ion beam testing with those obtained using a new picosecond pulsed laser facility," IEEE Trans. Nucl. Sci. 47, 539-544 (2000).
7. A. I. Chumakov, A. N. Egorov, O. B. Mavritskii, and A. V. Yanenko, "Evaluation of moderately focused laser irradiation as a method for simulating single-event effects," Russ. Microelectron. 33, 106 (2004).

8. A. V. Yanenko, A. I. Chumakov, A. A. Pechenkin, D. V. Savchenkov, A. S. Taraksin, and A. L. Vasil'ev, "Comparative analysis of tests of an electronic component base for resistance to the action of individual nuclear particles on laser simulators and ion accelerators," Spetstekh. Svyaz', Nos. 4-5 (2011).

9. A. I. Chumakov, "Possibilities and limitations of laser methods in assessing the sensitivity parameters of LSIs to the effects of exposure to heavy charged particles," Bezopasn. Inform. Tekhnol. 26 (3) (2019).

10. Yu. Ts. Oganessian, G. G. Gulbekyan, B. N. Gikal, and I. V. Kalagin, "Status report of the u 400 cyclotron at the FLNR JINR," in Proceedings of the APAC 2004 International Conference, Gyeongju, Korea, 2004, pp. 52-54.

11. E. V. Atkin, A. G. Voronin, A. D. Klyuev, V. V. Shumikhin, A. G. Voronin, I. A. Kudryuashov, D. M. Podorozhnyi, and A. Yu. Fedenko, "Read-out ASIC for microstrip detectors," Mikroelektronika 40, 57-63 (2011), Russ. Microelectron. 40, 52 (2011).

12. O. A. Vasilev, D. E. Karmanov, I. M. Kovalev, I. Kudryashov, A. Kurganov, A. Panov, D. Podorozhny, A. Slivin, E. Syresin, A. Turundaevsky, and G. Filatov, "Preliminary simulation results for the DPS-NICA project," Phys. Part. Nucl. Lett. 17, 871 (2020).

Translated by N. Semenova 